\title{
Sinfonia di soccorsi: un progetto memoriale di public history
}

Il seguente articolo descrive la realizzazione del progetto Sinfonia di soccorsi: un'iniziativa che dalla ricerca storica in archivio è arrivata a portare in scena nel corso di uno spettacolo teatrale la lettura di una selezione di lettere, telegrammi e comunicati arrivati al sindaco Renato Zangheri nei giorni e nei mesi successivi alla strage alla stazione di Bologna del 2 agosto 1980. La rabbia, il desiderio di aiutare e la semplice condivisione del lutto mostrata da persone provenienti da tutto il mondo, hanno potuto uscire dagli archivi e tornare a comunicare lo spirito di solidarietà che Bologna ricevette nel suo momento più difficile. Un bell'esempio di come la public history possa aiutare a riportare la storia tra le persone.

The following article describes the making of the project Sinfonia di soccorsi: an initiative which led up to a performance based on a selection of archived documents (letters, telegrams and condolences) sent from all over the world to the mayor of Bologna Renato Zangheri throughout the days and months following the attack on the Bologna railway station the 2nd of August 1980. The anger, the wish to help and the mourning expressed by people from all over the world could finally emerge from the archives and show the solidarity received by the city of Bologna during its most difficult moment. This whole project represents a good example of how public history can help to give back the real history to people. 


\section{II progetto}

Ogni anno, all'approssimarsi del 2 agosto, Bologna si prepara per un rituale molto importante ed estremamente carico di pathos. Fin dal 2 agosto del 1981 questo è un momento in cui la città si raccoglie, ricorda, soffre e afferma il suo esserci ancora, anche se con qualche cicatrice ogni tanto ignominiosamente riaperta. $\mathrm{La}$ città si mette in processione in un corteo silenzioso, dignitoso ma fiero, che accompagna i familiari delle vittime da Palazzo D'Accursio - qui vengono ricevuti in una cerimonia laica dalle istituzioni cittadine, locali e talvolta nazionali - fino alla stazione dove alle 10:25, durante un minuto di silenzio, si ricordano le ottantacinque vittime e si omaggiano i duecento feriti. La città accoglie le rappresentanze dei comuni di provenienza dei caduti, accoglie i familiari e mostra tutta la sua solidarietà così come fece in quel 2 agosto di trentotto anni fa.

Bologna meritò la medaglia civile conferitale il 3 luglio 1981 dal Presidente della Repubblica con la seguente motivazione:

A seguito del criminale attentato terroristico che sconvolse duramente la città, l'intera popolazione, pur emotivamente coinvolta, dava eccezionale prova di democratica fermezza e di civile coraggio, in una gara spontanea di solidarietà collaborava attivamente con gli organi dello stato, prodigandosi con esemplare slancio nelle operazioni di soccorso. Contribuiva così per la tempestività e l'efficienza, a salvare dalla morte numerose vite umane, suscitando il plauso e l'incondizionata ammirazione della Nazione.

Ma Bologna non fu lasciata sola. Da ogni parte d'Italia e da ogni parte del mondo molti espressero le proprie condoglianze, si offrirono di dare una mano o inviarono denaro. Una solidarietà che non conosceva frontiere, genuina e spontanea, che ha lasciato un segno indelebile nell'immaginario collettivo di chi visse quei giorni terribili, ma ha lasciato anche centinaia e centinaia di documenti che oggi costituiscono una delle voci più ricche del Fondo del Gabinetto del sindaco conservato presso l'Archivio storico comunale. Telegrammi, lettere, qualche foto, disegni di progetti artistici creati e donati al sindaco: un insieme di voci diverse, ma unite nel creare una vera e propria Sinfonia di soccorsi.

Con questo titolo si è voluto presentare lo spettacolo teatrale che il 1 agosto 2018 ha ridato voce a chi urlò la propria indignazione, a chi dignitosamente offrì la propria professionalità, il proprio denaro o addirittura il sangue, a chi semplicemente comunicava con dispiacere che non avrebbe potuto essere presente alla cerimonia funebre. Sinfonia di soccorsi è un progetto che parte almeno dal settembre 2017, quando come ogni anno l'Associazione dei familiari e la Regione Emilia Romagna, coadiuvati dalla professoressa Cinzia Venturoli, hanno cominciato a pensare a come si poteva celebrare il 2 agosto in un modo che andasse oltre la 
cerimonia ufficiale. Da alcuni anni infatti far uscire il 2 agosto dallo schema, pur importantissimo e dignitoso, del rito civile e istituzionale è diventato uno degli obiettivi principali dell'Associazione. Ciò viene fatto per ricordare le persone che morirono, chi venne ferito e chi prestò soccorso in un modo meno retorico e più vicino alle persone, in particolar modo a chi o non era ancora nato o era troppo piccolo per ricordare. La speranza è che così la strage continui a interrogare le persone e continui a ricordare loro che c'è una verità storica e giudiziaria che è stata raggiunta e va difesa da chi la vuole cancellare o storpiare, e c'è una verità storica e giudiziaria ancora da stabilire con certezza e per la quale bisogna lottare, come stanno lottando ogni mercoledì i familiari e l'associazione presso l'aula del Tribunale di Bologna in cui è in corso il processo a Gilberto Cavallini.

La realizzazione di questo progetto è stata il motivo principale per il quale il sottoscritto, studente del Master di public history dell'Università di Modena e Reggio Emilia, ha scelto di svolgere un percorso di tirocinio presso l'Associazione tra i familiari delle vittime della strage alla stazione di Bologna. Tale percorso ha permesso a un aspirante public historian di confrontarsi con gli aspetti organizzativi e realizzativi di un progetto culturale nato grazie al contributo dell'Assemblea legislativa della Regione Emilia Romagna, al lavoro di ricerca compiuto dalla storica Cinzia Venturoli e all'impegno del regista Matteo Belli. In base alle considerazioni riguardo a questa esperienza sono state realizzate una tesi di laurea e questo articolo.

\section{Fondo del Gabinetto del sindaco}

Il Fondo del Gabinetto del sindaco conservato presso l'Archivio storico comunale di Bologna rappresenta la partitura su cui si è poi sviluppato il progetto Sinfonia di soccorsi. Il suo contenuto è talmente vivo e significativo che la sua potenzialità espressiva può essere facilmente portata in scena. Si tratta infatti di telegram$\mathrm{mi}$, lettere, cartoline, resoconti, articoli di giornale che hanno la forza di attirare l'attenzione dello storico per la loro forte semplicità. Nonostante la presenza di numerosa corrispondenza ufficiale, la maggior parte dei documenti provengono da privati che in quell'occasione si sentirono di dover mostrare anche solo un piccolo gesto di solidarietà. Dalle semplici condoglianze, alle offerte di donazioni di denaro e sangue. Se si può azzardare un paragone, quello che ci si trova davanti consultando questo fondo archivistico di trentotto anni fa è la versione cartacea di quanto è avvenuto sui social network in occasione dei tragici eventi parigini del 7 gennaio e del 13 novembre 2015. Il desiderio della gente di comunicare la propria sofferenza, lo sconforto, in alcuni casi anche la propria rabbia e soprattutto 
la propria solidarietà è molto simile; tuttavia esistono alcune piccole, ma significative differenze, rappresentate da quella serie di gesti necessari per inviare una lettera o un telegramma, che li rendono automaticamente più intimi e profondi di un tweet. Si tratta inoltre di documenti che per il modo stesso in cui sono scritti assumono un valore archetipico del senso di umanità che le persone mostrano in queste tragiche circostanze.

Tra la notevole mole di documenti, ogni lettera, ogni telegramma, ogni donazione ha una sua specifica storia e significato. Per questo motivo fin dal primo giorno di ricerca effettuata in archivio si è deciso di procedere a una selezione ragionata. $\mathrm{Si}$ è voluto in particolare mettere alcuni filtri che permettessero una scelta più utile ai fini dello spettacolo, tenendo conto dell'interesse del pubblico e dando risalto alla voce dei tanti sconosciuti che espressero solidarietà. Ovviamente anche i comunicati ufficiali delle ambasciate, dei consolati, dei comuni e del mondo istituzionale sono stati analizzati, e i più significativi selezionati. Ma classificando per temi e per soggetti i documenti prescelti, si sono potuti costruire nuclei omogenei di fonti e facilitare la confezione di un'antologia, pubblicata col titolo Sinfonia di soccorsi. La solidarietà e il dolore del mondo al sindaco Zangheri grazie al contributo dell'Assemblea regionale e dell'Archivio storico comunale.

\section{Le prove dello spettacolo}

Lo spettacolo-che secondo la terminologia teatrale è una composizione drammaturgica in forma di fonologo-è stato realizzato grazie al regista Matteo Belli e agli attori: Lucrezia Bruchi, Matteo Baschieri, Elisa Calderara, Roberto Di Lao, Benedetta Dora Mannina, Lorenzo Monti, Simonetta Scappini, Tiziana Scimone, Domenico Troncato e Samantha Zanarini. Nella prima fase di preparazione il regista e gli attori si sono soffermati maggiormente sugli aspetti tecnici della recitazione all'interno di un seminario di formazione teatrale con frequenza obbligatoria, funzionale all'elaborazione di un linguaggio comune e alla condivisione di saperi e pratiche di lavoro differenti. Un obiettivo che-a parere di chi scrivecoincide totalmente con la public history e i suoi principi di comunicazione della storia, interazione con e per il pubblico (in questo caso diviso su due livelli: gli attori nella fase preparatoria e il pubblico nella messa in scena) e compresenza di differenti professionalità, al fine ultimo di creare un ponte tra la disciplina storica e la società [Ridolfi 2017; Bertucelli 2017]. In base a queste scelte si è potuto creare un gruppo molto affiatato di attori di età differenti - dai 20 ai 53 anni che al momento del confronto coi documenti d'archivio apparivano consapevoli dell'importanza sociale, civile e storica di quanto stavano per mettere in scena. 
Un aspetto che si ritiene fondamentale ricordare, perché altrimenti Sinfonia di soccorsi potrebbe confondersi con altre centinaia di produzioni teatrali basate sulla narrazione di fatti storici, sui quali gli attori sono spesso solo vagamente informati. Il copione dello spettacolo è stato creato da Matteo Belli attraverso una sapiente opera di selezione dei documenti-necessaria fra l'altro per la resa scenica-che sono stati poi disposti nel testo senza alcun tipo di rimaneggiamento testuale. Segno di comprensione dell'importanza dell'integrità del documento da un punto di vista storico e quindi testimonianza della capacità della public history di saper diffondere la metodologia della disciplina anche al di fuori della cerchia degli storici.

\section{Considerazioni finali}

Lo spettacolo è stato messo in scena la sera dell' 1 agosto 2018 presso piazza Renzo Imbeni, alla presenza di un pubblico numeroso. Si è trattato di un successo, come riportato anche dai giornali e dalle televisioni locali, per tutti coloro che hanno a cuore la memoria della strage, la città di Bologna, il senso civico e la storia. Grazie all'impegno dell'ufficio stampa della Regione e a Lepida Tv, sul sito dell'Assemblea legislativa è possibile scaricare il pdf dell'antologia dei documenti e vedere per intero lo spettacolo. La buona riuscita di questa iniziativa mostra ancora una volta come laddove la memoria, ma ancor più la storia, riesce a trovare una modalità di approccio con il pubblico che vada al di là dei classici canali di divulgazione, essa risvegli l'interesse delle persone, le spinga ad approfondire e a non prendere più in maniera acritica i tanti falsi storici. Soprattutto rispetto a un tema così poco trattato nelle scuole e quindi tendenzialmente sempre più dimenticato, occorre che la comunità degli storici si faccia carico di intervenire per contrastare la diffusione di semplificazioni o di vere e proprie inesattezze. Solo così si possono colmare le lacune e costruire dei veri percorsi di militanza civica come baluardo contro l'approssimazione e la banalizzazione. Occorre però che lo storico sia in grado di parlare a un pubblico composto non esclusivamente da esperti. Lo storico deve scendere dalla torre d'avorio e rivendicare uno spazio pubblico in cui confrontarsi con la cittadinanza, in cui diffondere una metodologia di lavoro non sindacabile e in cui impari a rispondere a quella domanda di storia che oggi è più che mai presente nella società, ma a cui troppo spesso rispondono professionisti che storici non sono. Per questo è importante valorizzare e diffondere esperienze e metodologie della public history, disciplina che grazie alla sua apertura e alla sua elasticità permette di fare storia con il pubblico e per il pubblico. 
A testimoniare l'efficacia di questa modalità di lavoro vorrei concludere con una breve digressione riguardo a un episodio avvenuto nel corso delle prove. L'unica lettera letta in forma integrale nello spettacolo è quella che tra gli addetti ai lavori è nota come la "lettera di una ragazza qualsiasi", perché proprio così è firmata. Si tratta di una testimonianza molto accorata e dal forte impatto emotivo di una giovane ragazza molto probabilmente reduce dal movimento del '77, preoccupata come molti suoi coetanei per il cosiddetto "riflusso", sfiduciata dalla sinistra tradizionale, ma consapevole dell'assurda inutilità della lotta armata e della ricerca dei paradisi artificiali delle droghe. Nelle sue parole si ritrova il dramma di una generazione composta da chi aveva sperato in un'ipotesi rivoluzionaria e di fronte all'elaborazione di una sconfitta politica, si trova a dover fare i conti con l'ennesimo episodio di violenza cieca e insensata. Di fronte a tutto ciò la rabbia monta, ma viene smorzata di fronte alla reazione civile, dignitosa, ma estremamente risoluta della città di Bologna il giorno dei funerali il 6 agosto 1980. Per la fiducia che questa città le infonde la ragazza qualsiasi ringrazia il sindaco Zangheri, che nel recente passato era stato un nemico per chi aveva fatto parte del movimento del' 77 .

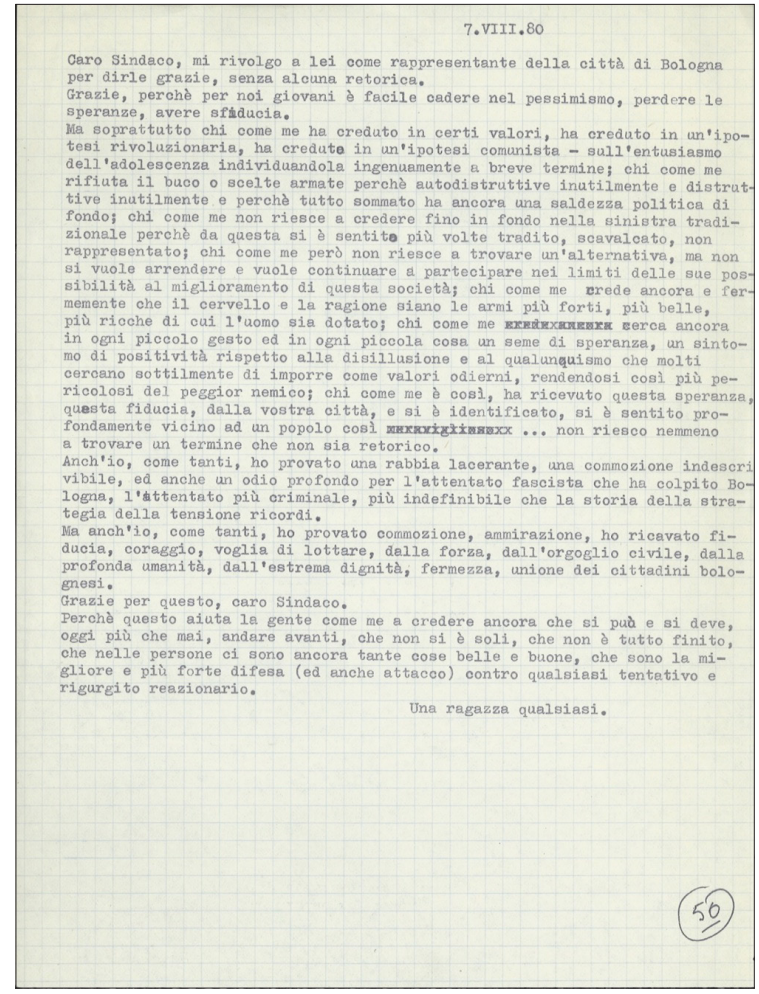

Archivio storico del Comune di Bologna, Gabinetto del sindaco, Strage alla stazione, Lettera di una ragazza qualsiasi, 7 agosto 1980.
Sono parole vibranti e piene di spunti interessantissimi, che raccontano con immagini incredibilmente dirette quegli anni in poche frasi. È un testo che sembra adattarsi totalmente allo spirito dello spettacolo e al raccontare la solidarietà umana espressa al sindaco Zangheri e a Bologna in quei giorni. Il compito di leggerla era quindi particolarmente gravoso perché Matteo Belli voleva che il testo venisse interpretato con un certo pathos, che doveva rievocare la disillusione ma anche la speranza in un mondo migliore e in un ritorno al collettivo come reazione all'individualismo montante 
che vengono espressi nella conclusione. Si tratta di concetti che sicuramente vennero scritti con un certo spirito militante e quindi da leggere in quel particolare modo, che poteva essere facilmente immaginato da chi ha vissuto in quegli anni, o da chi li ha studiati da un punto di vista storico, molto meno da chi non c'era. Nonostante questo il regista ha voluto affidare la lettura a una delle attrici più giovani. Ciò rappresentava una sfida poiché si trattava di rendere sì più credibile la lettura affidandola a una coetanea dell'autrice, ma parzialmente anche di un azzardo essendo l'attrice in questione parzialmente digiuna di conoscenza storica di quel periodo. Dopo un paio di prove buone ma non esattamente convincenti, Belli ha deciso di compiere una sorta di esegesi del testo per la giovane attrice, sottolineando in modo molto efficace i significati di quello che stava leggendo, usando le parole del testo e affiancandovi alcune immagini moderne (come per esempio lo sbigottimento generale del post 11 settembre o dei giorni successivi alle stragi di "Charlie Hebdo" e del Bataclan) per rendere più accessibile il sentimento con cui la "ragazza qualsiasi" scrisse la sua lettera. Il risultato è stato sorprendente e al momento dell'ultima prova l'energia scaturita dalle parole è stata tale da commuovere la giovane attrice che le leggeva.

\section{Bibliografia}

Assemblea legislativa della Regione Emilia Romagna (a cura di) 2018, Sinfonia di Soccorsi - La solidarietà e il dolore del mondo al sindaco Zangheri, Bologna: centro stampa RER

Bertucelli L. 2017, La Public History in Italia. Metodologie, pratiche, obiettivi, in Bertella Farnetti P., Bertucelli L., Botti A. (a cura di) 2017, Public History. Discussioni e pratiche, Milano: Mimesis, pp 75-96

Ridolfi M. 2017, Non solo "uso pubblico della storia": verso una Public History italiana, in Bertella Farnetti P., Bertucelli L., Botti A. (a cura di) 2017, Public History. Discussioni e pratiche, Milano: Mimesis, pp 107-130

\section{Risorse on line}

Sinfonia di soccorsi (Bologna, 1 agosto 2018)

https://cronacabianca.eu/tv/video/sinfonia-di-soccorsi-lo-spettacolo/

Regione Emilia Romagna, Assemblea legislativa-2 agosto, il portale https://www.assemblea.emr.it/cantiere-due-agosto/sinfonia-di-soccorsi 